\title{
Non-Markovian quantum input-output networks
}

\author{
Jing Zhang, ${ }^{1,2,3, \text {, }}$ Yu-xi Liu, ${ }^{4,2,3}$ Re-Bing Wu, ${ }^{1,2,3}$ Kurt Jacobs, ${ }^{5,3}$ and Franco Nori ${ }^{3,6}$ \\ ${ }^{1}$ Department of Automation, Tsinghua University, Beijing 100084, P. R. China \\ ${ }^{2}$ Center for Quantum Information Science and Technology, TNList, Beijing 100084, P. R. China \\ ${ }^{3}$ Advanced Science Institute, RIKEN, Wako-shi, Saitama, 351-0198, Japan \\ ${ }^{4}$ Institute of Microelectronics, Tsinghua University, Beijing 100084, P. R. China \\ ${ }^{5}$ Department of Physics, University of Massachusetts at Boston, Boston, MA 02125, USA \\ ${ }^{6}$ Physics Department, The University of Michigan, Ann Arbor, Michigan 48109-1040, USA
}

(Dated: August 24, 2012)

\begin{abstract}
Quantum input-output response analysis is a useful method for modeling the dynamics of complex quantum networks, such as those for communication or quantum control via cascade connections. Non-Markovian effects have not yet been studied in such networks. Here we extend the Markovian input-output network formalism developed in optical systems to non-Markovian cascaded networks which can be used, e.g., to analyze the input-output response of mesoscopic quantum networks. We use this formalism to explore the behavior of superconducting qubit networks, where we examine the effect of finite cavity bandwidths. We also discuss its application to open- and closed-loop control networks, and show how these networks create effective Hamiltonians for the controlled system.

PACS numbers: 03.65.Yz,42.50.Lc,03.67.-a
\end{abstract}

\section{INTRODUCTION}

There has been tremendous progress in the last few years in experimental efforts to realize quantum networks 1] in various mesoscopic systems. These systems include photonic crystals [2], ion traps [3], and superconducting circuits [4], which also advancesrelated fields such as quantum simulation (for recent reviews, see, e.g., Ref. [5]). The input-output formalism of Gardiner and Collet [6, 7] is a useful tool for analyzing such networks. In fact, using the input-output response to analyze or even modify the dynamics is a standard method in engineering called system synthesis. Up until now, system synthesis for quantum networks has only been studied for Markovian systems. Quantum input-output theory itself has also mainly been limited to the Markovian regime, although it was developed for quantum systems about twenty years ago [6]. It was extended to the nonMarkovian systems only quite recently [8] .

In the existing literature [6, 7], quantum input-output theory is mainly applied to optical systems, in which the coupling between the system and its environment is weak and the correlation time (the "memory" of the environment) is small compared with the characteristic timescale of the system dynamics. Under the Markovian assumption, the quantum input-output formalism [6] was extended to cascaded systems [7], and has been used to study quantum feedforward and feedback networks [911]. Markovian quantum input-output networks can be described using two alternative formulations: the Hudson-Parthasarity formalism in the Schrödinger picture [12]; and the quantum transfer function formalism in the Heisenberg picture [13, 14]. The general algebraic

*Electronic address: jing-zhang@mail.tsinghua.edu.cn structure of such systems has been well studied in the language of quantum Wiener and Poisson processes and quantum Ito rules [12].

Although the Markovian assumption is reasonable when considering optical network components, environments in mesoscopic solid-state systems can have correlations on much longer timescales [15 18]. Examples of this are the nuclear spin bath that couples to electron spins in quantum dots [19], and the $1 / f$ noise that affects Josephson-Junction qubits 20]. It has also been suggested that the damping and decoherence of nanomechanical resonators are due to coupling to a small number of two-level systems [21], which can be expected to induce significant non-Markovian dynamics. In addition, any classical noise with a sufficiently narrow band generates non-Markovian evolution. There has been increasing interest in recent years in non-Markovian open quantum systems, and a number of analytical approaches have been devised to describe them. These include the projection-operator partitioning technique [22], the nonMarkovian quantum trajectory approach [23], and very recently a non-Markovian input-output formalism [8].

In this paper we extend the non-Markovian inputoutput theory to cascaded quantum networks, providing a recipe for obtaining non-Markovian input-output equations for the description of any such network. Naturally, this formalism reduces to the standard input-output network formalism in the Markovian limit. Although the non-Markovian input-output relation has been derived in Ref. [8], a quantum measurement is imposed on the output field in Ref. [8] and the system dynamics is described by the quantum state diffusion equation. Such a formalism cannot be extended to describe a non-Markovian cascade system, because quantum coherence in the output field is deteriorated by the measurement. In our formalism, without introducing measurements, the system dynamics is described by a non-Markovian quantum 
stochastic differential equation and a perturbative master equation which can be naturally extended to a nonMarkovian network.

In developing our non-Markovian formalism, we will keep the weak-noise (weak-coupling) approximation, as this is the appropriate regime for implementing quantum technologies (for recent reviews, see, e.g., Ref. 24]), such as information processing and metrology. Here, we go beyond the Markovian approximation by allowing the coupling to the bath to have an arbitrary frequency dependence. This allows one to describe noise with any frequency profile, and should provide a good model for a wide range of non-Markovian environments. Interestingly, the resulting non-Markovian network formalism is exact for all couplings. However, in order to perform calculations for nonlinear systems, one must transform the Heisenberg equations of the input-output formalism to non-Markovian master equations, and this requires further approximations. Here we do this at the simplest level of approximation, by deriving the corresponding master equation to second-order in perturbation theory, using the standard Born approximation [22]. Nevertheless, more sophisticated techniques exist for obtaining non-Markovian master equations, and it would be an interesting avenue for future work to examine how these can be used to obtain master equations for nonMarkovian cascaded networks. We note that for linear systems the Heisenberg equations of the input-output formalism can be used to obtain exact results. This is especially useful in some cases [25], when the secondorder perturbative master equation fails to behave correctly [26]. We expect the network formalism we develop to be useful in describing a range of mesoscopic systems, such as coupled-cavity arrays in photonic crystals 27, 28, and nonlinear resonator and qubit networks in solid-state circuits [29 32]. The formalism can also be applied to quantum feedback control networks 33 39] in solid-state systems 40 47].

This paper is organized as follows: in Sec. II, we briefly review the Markovian input-output formalism so that this can be easily compared to the non-Markovian case. In Sec. III, we use an alternative method to derive the non-Markovian input-output relations in Ref. [8], and obtain the dynamical equation for the system such that it can be easily used for networks. We derive these here as a natural extension of the original Collett-Gardiner quantum input-output theory. In Sec. IV, we derive the input-output relations for more complex non-Markovian quantum cascade networks. We then apply these general results to two examples: a simple non-Markovian oscillator, and a network of two superconducting charge qubits interacting with Lorentz noises. We also apply the formalism to an open-loop and a closed-loop control networks, showing how these networks create effective Hamiltonians for the controlled system. The conclusions and prospects for future work are discussed in Sec. V.

\section{BRIEF REVIEW OF INPUT-OUTPUT THEORY OF MARKOVIAN SYSTEMS}

Here we summarize the standard Gardiner-Collet input-output formalism [6]. The basic model is a quantum system interacting with a bath, where the bath consists of the modes of an electromagnetic field, or equivalently a continuum of harmonic oscillators. The Hamiltonian for the system and bath is

$$
H=H_{S}+H_{B}+H_{\mathrm{int}},
$$

with

$$
\begin{aligned}
H_{B} & =\int_{-\infty}^{+\infty} \omega b^{\dagger}(\omega) b(\omega) d \omega \\
H_{\mathrm{int}} & =i \int_{-\infty}^{+\infty}\left[\kappa(\omega) b^{\dagger}(\omega) L-\text { h.c. }\right] d \omega,
\end{aligned}
$$

where $b^{\dagger}(\omega)$ and $b(\omega)$ are the creation and annihilation operators of the bath mode with frequency $\omega$, which satisfy

$$
\left[b(\omega), b^{\dagger}(\tilde{\omega})\right]=\delta(\omega-\tilde{\omega}) .
$$

In the above, $H_{S}$ is the free Hamiltonian of the system. The bath mode with frequency $\omega$ interacts with the system via the system operator $L$ and the coupling strength $\kappa(\omega)$. Hereafter we set $\hbar=1$. The total Hamiltonian $H$ can be reexpressed in the interaction picture as

$$
\begin{aligned}
H_{\mathrm{eff}} & =\exp \left(i H_{B} t\right)\left(H_{S}+H_{\mathrm{int}}\right) \exp \left(-i H_{B} t\right) \\
& =H_{S}+i \int_{-\infty}^{+\infty}\left[\kappa(\omega) e^{i \omega t} b^{\dagger}(\omega) L-\text { h.c. }\right] d \omega .
\end{aligned}
$$

If the coupling strength is constant for all frequencies, so that

$$
\kappa(\omega)=\sqrt{\frac{\gamma}{2 \pi}}
$$

then the dynamics of the system will become Markovian. This is the Markovian approximation. The Hamiltonian $H_{\text {eff }}$ is now given by

$$
H_{\mathrm{eff}}=H_{S}+i \sqrt{\gamma}\left[b_{\mathrm{in}}^{\dagger}(t) L-L^{\dagger} b_{\mathrm{in}}(t)\right],
$$

where

$$
b_{\text {in }}(t)=\frac{1}{\sqrt{2 \pi}} \int_{-\infty}^{+\infty} e^{-i \omega t} b(\omega) d \omega
$$

is the Fourier transform of the bath modes, and is the time-varying input field that is fed into the system [48] (see Fig. 1). In the Heisenberg picture, the system operator $X(t)$ satisfies the following quantum stochastic differential equation (QSDE)

$$
\begin{aligned}
\dot{X}= & -i\left[X, H_{S}\right]+\frac{\gamma}{2}\left\{L^{\dagger}[X, L]+\left[L^{\dagger}, X\right] L\right\} \\
& +\sqrt{\gamma}\left\{b_{\text {in }}\left[L^{\dagger}, X\right]+[X, L] b_{\text {in }}^{\dagger}\right\} .
\end{aligned}
$$




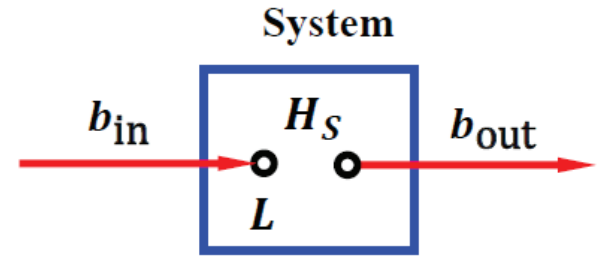

FIG. 1: (Color online) Schematic diagram of the Markovian input-output system.

If the input field is in a $b_{\text {in }}(t)$ vacuum state, and we trace it out, we can re-express the system dynamics in the Schrödinger picture as the following master equation

$$
\dot{\rho}=-i\left[H_{S}, \rho\right]+\gamma\left(L \rho L^{\dagger}-\frac{1}{2} L^{\dagger} L \rho-\frac{1}{2} \rho L^{\dagger} L\right) .
$$

Finally, if $b_{\text {out }}(t)$ is the field after it has interacted with the system, and is now out of it, then one has the relation

$$
b_{\text {out }}(t)=b_{\text {in }}(t)+\sqrt{\gamma} L(t) .
$$

This is the Markovian input-output relation.

\section{INPUT-OUTPUT THEORY OF NON-MARKOVIAN SYSTEMS}

\section{A. General theory}

To derive the input-output relation for a general nonMarkovian quantum system (see Fig. 2), we rewrite the Hamiltonian $H_{\text {eff }}$ in Eq. (4) as

$$
\begin{aligned}
H_{\mathrm{eff}} & =H_{S}+i\left\{\left[\int_{-\infty}^{+\infty} \kappa(\tau-t) b_{\mathrm{in}}^{\dagger}(\tau) d \tau\right] L-\text { h.c. }\right\} \\
& =H_{S}+i\left[\tilde{b}_{\mathrm{in}}^{\dagger}(t) L-L^{\dagger} \tilde{b}_{\mathrm{in}}(t)\right],
\end{aligned}
$$

where

$$
\kappa(t)=\frac{1}{\sqrt{2 \pi}} \int_{-\infty}^{+\infty} \exp (-i \omega t) \kappa(\omega) d \omega
$$

is the Fourier transform of the coupling strength $\kappa(\omega)$. The input field that interacts directly with the system is now

$$
\tilde{b}_{\text {in }}(t)=\int_{-\infty}^{+\infty} \kappa(t-\tau) b_{\text {in }}(\tau) d \tau
$$

and satisfies the new commutation relation

$$
\left[\tilde{b}_{\text {in }}(t), \tilde{b}_{\text {in }}^{\dagger}(\tilde{t})\right]=\gamma(t-\tilde{t}),
$$

where

$$
\gamma(t-\tilde{t})=\int_{-\infty}^{+\infty} \kappa^{*}(t-\tau) \kappa(\tilde{t}-\tau) d \tau
$$

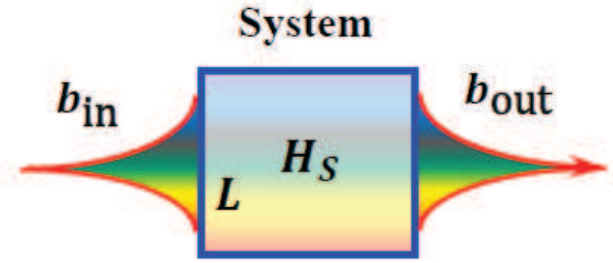

FIG. 2: (Color online) Schematic diagram of the nonMarkovian input-output system. The input field is dispersed when it interacts with the system, and the modes with different frequencies in the input field are coupled to the system with different coupling strengths.

We can now proceed to derive the Heisenberg stochastic differential equations for the evolution of the system:

$$
\begin{aligned}
\dot{X}= & -i\left[X, H_{S}\right]+\int_{0}^{t}\left\{\gamma(t-\tau) L^{\dagger}(\tau)[X(t), L(t)]\right. \\
& \left.+\gamma^{*}(t-\tau)\left[L^{\dagger}(t), X(t)\right] L(\tau)\right\} d \tau \\
& +\left\{\tilde{b}_{\text {in }}(t)\left[L^{\dagger}, X\right]+[X, L] \tilde{b}_{\text {in }}^{\dagger}(t)\right\} .
\end{aligned}
$$

The non-Markovian input-output relation becomes

$$
b_{\text {out }}(t)=b_{\text {in }}(t)+\int_{0}^{t} \kappa(t-\tau) L(\tau) d \tau .
$$

Note so far that no further assumptions have been made, based on the Hamiltonian in Eq.(11). Thus our nonMarkovian network formalism will give a more precise description of non-Markovian systems with environment's coupling that have an arbitrary frequency-dependence. However, as noted in the introduction, for non-linear networks we must often resort to the Schrödinger picture to perform calculations.

To obtain the second-order perturbative master equation, one averages over the vacuum input field $b_{\text {in }}$, which we will take to be in the vacuum state, and uses the Born approximation. The perturbative master equation that corresponds to Eq. (16) is

$$
\begin{aligned}
\dot{\rho}= & -i\left[H_{S}, \rho\right]+\int_{0}^{t}\left\{\gamma(t-\tau)\left[L \rho(\tau), L_{\mathrm{H}_{\mathrm{S}}}^{\dagger}(\tau-t)\right]\right. \\
& \left.+\gamma^{*}(t-\tau)\left[L_{\mathrm{H}_{\mathrm{S}}}(\tau-t), \rho(\tau) L^{\dagger}\right]\right\} d \tau,
\end{aligned}
$$

where

$$
L_{\mathrm{H}_{\mathrm{S}}}(t)=\exp \left(i H_{S} t\right) L \exp \left(-i H_{S} t\right) .
$$

We give the details of the derivations of Eqs. (16) and (18), and output equation (17) in Appendix A

Remark 1: The input-output relation, i.e., Eq.(17), coincides with Diosi's non-Markovian input-output equation (Eq. (10) in Ref. [8]). However, the dynamics of the input-output system in Ref. [8] is described by 


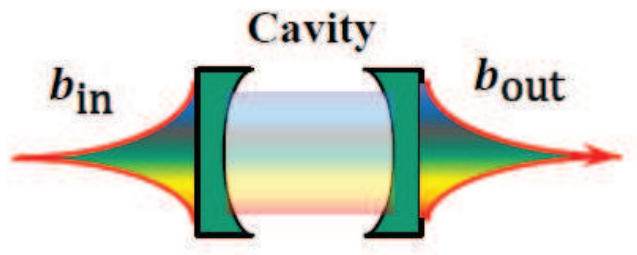

FIG. 3: (Color online) Schematic diagram of the linear nonMarkovian cavity.

the quantum state diffusion equation after introducing a heterodyne measurement on the output field. Such a formalism cannot be extended to derive the dynamics of more complex non-Markovian networks, because quantum effects of the input-output system have been reduced by the quantum measurements. In our formalism, without introducing measurement, the system dynamics is described by the non-Markovian quantum stochastic differential equation (16) and the perturbative master equation (18). Such a formalism can be more naturally extended to non-Markovian cascade networks.

Remark 2: In the Markovian limit, where $\kappa(\omega)=\sqrt{\gamma / 2 \pi}$, we have $\kappa(t)=\sqrt{\gamma} \delta(t)$, and $\gamma(t-\tilde{t})=\gamma \delta(t-\tilde{t})$. It can be easily verified that Eq. (16) reduces to the quantum stochastic differential equation (8) and the output equation (17) reduces to Eq. (10).

\section{B. Example: Single-mode cavity}

For a single-mode cavity coupled to an external input field (see Fig. 3), the system Hamiltonian $H_{S}$ and dissipation operator $L$ are given by $H_{S}=\omega_{0} a^{\dagger} a$ and $L=a$, where $\omega_{0}, a\left(a^{\dagger}\right)$ are respectively the frequency and the annihilation (creation) operator of the cavity mode. The mode has an arbitrary non-Markovian coupling to the field modes outside the cavity. The quantum stochastic differential equation for a cavity operator $X$ is then

$$
\begin{aligned}
\dot{X}= & -i\left[X, \omega_{0} a^{\dagger} a\right]+\int_{0}^{t}\left\{\gamma(t-\tau) a^{\dagger}(\tau)[X(t), a(t)]\right. \\
& \left.+\gamma^{*}(t-\tau)\left[a^{\dagger}(t), X(t)\right] a(\tau)\right\} d \tau \\
& +\left\{\tilde{b}_{\text {in }}\left[L^{\dagger}, X\right]+[X, L] \tilde{b}_{\mathrm{in}}^{\dagger}\right\} .
\end{aligned}
$$

Let us define the normalized position and momentum operators for the cavity as $q=\left(a+a^{\dagger}\right) / \sqrt{2}, p=$ $\left(-i a+i a^{\dagger}\right) / \sqrt{2}$. Collecting these into a single vector, $\vec{x}=(q, p)^{T}$, we may write the equations of motion for these operators as

$$
\begin{aligned}
\dot{\vec{x}}= & A_{0} \vec{x}+A_{1} \int_{0}^{t} \gamma(t-\tau) \vec{x}(\tau) d \tau \\
& +A_{1}^{*} \int_{0}^{t} \gamma^{*}(t-\tau) \vec{x}(\tau) d \tau+B \tilde{b}_{\mathrm{in}}+B^{*} \tilde{b}_{\mathrm{in}}^{\dagger},
\end{aligned}
$$

where

$$
A_{0}=\left(\begin{array}{cc}
0 & \omega_{0} \\
-\omega_{0} & 0
\end{array}\right), \quad A_{1}=\frac{1}{2}\left(\begin{array}{cc}
-1 & i \\
-i & -1
\end{array}\right) .
$$

and $B=(-1, i)^{T} / \sqrt{2}$.

To solve the dynamical equation (21), we use the Laplace transform

$$
O(s)=\int_{0}^{\infty} \exp (-s t) O(t) d t
$$

to transform the differential equation to an algebraic equation in the frequency domain. In this domain the solution becomes

$\vec{x}(s)=\frac{\left(\begin{array}{cc}d(s) & \omega_{0} \\ -\omega_{0} & d(s)\end{array}\right)}{\sqrt{2} \Delta(s)}\left(\begin{array}{l}-\kappa(s) b_{\text {in }}(s)-\kappa^{*}(s) b_{\text {in }}^{\dagger}(s) \\ i \kappa(s) b_{\text {in }}(s)-i \kappa^{*}(s) b_{\text {in }}^{\dagger}(s)\end{array}\right)$,

where

$$
\Delta(s)=d^{2}(s)+\omega_{0}^{2}, \quad d(s)=s+\gamma(s) / 2,
$$

and $\kappa(s), \vec{x}(s), b_{\text {in }}(s), b_{\text {in }}^{\dagger}(s), \gamma(s)$ are the Laplace transforms of $\kappa(t), \vec{x}(t), b_{\text {in }}(t), b_{\text {in }}^{\dagger}(t), \gamma(t)$. We can also obtain the following input-output relation in the frequency domain

$$
b_{\text {out }}(s)=\frac{s+\gamma(s) / 2-\kappa^{2}(s)+i \omega_{0}}{s+\gamma(s) / 2+i \omega_{0}} b_{\text {in }}(s) .
$$

Note that this result is exact as far as the frequency dependence of the coupling to the bath is concerned. Because the system is linear we can obtain results without deriving a master equation. This input-output formula shows exactly how the coupling profile applies a low-pass filter to the input field to produce the output field.

Remark 3: Let us consider a Markovian cavity with damping rate $\gamma$, then we have $\kappa(s)=\sqrt{\gamma}, \gamma(s)=\gamma$, from which we can obtain the traditional input-output relation for a lossy cavity from Eq. (25) (see Eq. (45) in Ref. [14])

$$
b_{\text {out }}(s)=\frac{s-\gamma / 2+i \omega_{0}}{s+\gamma / 2+i \omega_{0}} b_{\text {in }}(s)
$$




\section{NON-MARKOVIAN QUANTUM NETWORKS}

\section{A. General theory: quantum cascade systems}

To derive the input-output relation for complex nonMarkovian networks, we should first study the dynamics of a system composed of two cascade-connected subsystems, also known as the "series product" of two subsystems [9]. The Hamiltonian of two cascaded subsystems, depicted in Fig. 4 can be expressed as

$$
\begin{aligned}
H_{\mathrm{eff}} & =H_{1}+i\left\{\left[\int_{-\infty}^{+\infty} \kappa_{1}(\tau-t) b_{1, \text { in }}^{\dagger}(\tau) d \tau\right] L_{1}-\text { h.c. }\right\} \\
& +H_{2}+i\left\{\left[\int_{-\infty}^{+\infty} \kappa_{2}(\tau-t) b_{2, \text { in }}^{\dagger}(\tau) d \tau\right] L_{2}-\text { h.c. }\right\},
\end{aligned}
$$

where $H_{i=1,2}$ and $L_{i=1,2}$ are the free Hamiltonian and dissipation operator of the $i$-th subsystem; and $\kappa_{i}(t)$ is the corresponding coupling strength between the $i$-th subsystem and the $i$-th input field. If we omit the time delay for the quantum field transmitting between the two inputoutput components, then we have

$$
b_{2, \text { in }}(t)=b_{1, \text { out }}(t)=b_{1, \text { in }}(t)+\int_{0}^{t} \kappa_{1}(t-\tau) L_{1}(\tau) d \tau .
$$

Substituting Eq. (28) into Eq. (27), we have

$$
H_{\mathrm{eff}}=H_{1}+H_{2}+H_{12}+i \sum_{j=1,2}\left[\tilde{b}_{j, \text { in }}^{\dagger} L_{j}-L_{j}^{\dagger} \tilde{b}_{j, \text { in }}\right]
$$

where

$$
H_{12}=-i \int_{0}^{t}\left[\gamma_{12}^{\theta}(\tau-t) L_{2} L_{1}^{\dagger}(\tau)-\text { h.c. }\right] d \tau
$$

is the interaction Hamiltonian between the two subsystems introduced by the transmitting field; the parameter $\gamma_{12}^{\theta}(\tau-t)$ is defined by

$$
\gamma_{12}^{\theta}(t-\tilde{t})=\int_{-\infty}^{+\infty} \kappa_{1}^{*}(\tau-t) \kappa_{2}(\tau-\tilde{t}) \theta(t-\tau) d \tau
$$

and $\theta(t)$ is the step function

$$
\theta(t)=\left\{\begin{array}{l}
1, t \geq 0 \\
0, t<0
\end{array}\right.
$$

The two equivalent non-Markovian input fields that interact directly with the two subsystems via the dissipation operators $L_{1}$ and $L_{2}$ are defined as

$$
\tilde{b}_{l, \text { in }}(t)=\int_{-\infty}^{+\infty} \kappa_{l}(t-\tau) b_{\text {in }}(\tau) d \tau
$$

and these satisfy the following commutation relation

$$
\left[\tilde{b}_{l, \text { in }}(t), \tilde{b}_{r, \text { in }}^{\dagger}\right]=\gamma_{l r}(t-\tilde{t}),
$$

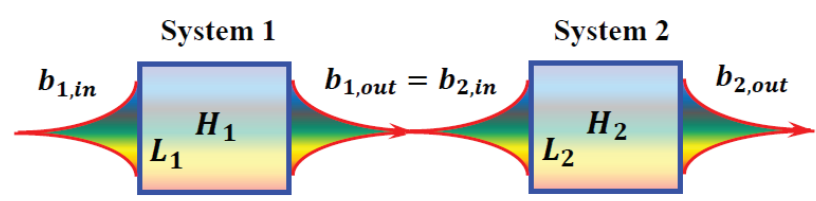

FIG. 4: (Color online) Schematic diagram of the nonMarkovian quantum cascade system. The output from the first subsystem is fed into the input of the second subsystem.

where

$$
\gamma_{l r}(t-\tilde{t})=\int_{-\infty}^{+\infty} \kappa_{r}^{*}(t-\tau) \kappa_{l}(\tilde{t}-\tau) d \tau .
$$

The dynamics of the total system can then be expressed as the following quantum stochastic differential equation

$$
\begin{aligned}
\dot{X}= & \sum_{l, r=1}^{2} \int_{0}^{t}\left\{\gamma_{l r}(t-\tau) L_{l}^{\dagger}(\tau)\left[X(t), L_{r}(t)\right]\right. \\
& \left.+\gamma_{l r}^{*}(t-\tau)\left[L_{r}^{\dagger}(t), X(t)\right] L_{l}(\tau)\right\} d \tau \\
& -i\left[X, H_{S}\right]+\sum_{l=1}^{n}\left\{\tilde{b}_{l, \text { in }}\left[L_{l}^{\dagger}, X\right]+\left[X, L_{l}\right] \tilde{b}_{l, \text { in }}^{\dagger}\right\},
\end{aligned}
$$

where $H_{S}=H_{1}+H_{2}+H_{12}$. The input-output equation of the cascade system can be expressed as

$$
\begin{aligned}
b_{\text {out }}(t)= & b_{\text {in }}(t)+\int_{0}^{t} \kappa_{1}(t-\tau) L_{1}(\tau) d \tau \\
& +\int_{0}^{t} \kappa_{2}(t-\tau) L_{2}(\tau) d \tau .
\end{aligned}
$$

Remark 4: In the Markovian limit, we have $\kappa_{l}(t)=$ $\sqrt{\gamma_{l}} \delta(t), \gamma_{l r}^{\theta}(t-\tilde{t})=\gamma_{l r}(t-\tilde{t})=\sqrt{\gamma_{l} \gamma_{r}} \delta(t-\tilde{t})$, and $\tilde{b}_{l, \text { in }}=b_{\text {in }}$. Thus, the dynamical equation (37) can be re-expressed as

$$
\begin{aligned}
\dot{X}= & -i\left[X, H_{S}\right]+\frac{1}{2}\left\{L^{\dagger}[X, L]+\left[L^{\dagger}, X\right] L\right\} \\
& b_{\text {in }}\left[L^{\dagger}, X\right]+[X, L] b_{\text {in }}^{\dagger},
\end{aligned}
$$

where

$$
\begin{aligned}
H_{S} & =H_{1}+H_{2}+\frac{i \sqrt{\gamma_{1} \gamma_{2}}}{2}\left(L_{1}^{\dagger} L_{2}-L_{1} L_{2}^{\dagger}\right) \\
L & =\sqrt{\gamma_{1}} L_{1}+\sqrt{\gamma_{2}} L_{2},
\end{aligned}
$$

and output equation (40) can be rewritten as

$$
b_{\text {out }}(t)=b_{\text {in }}(t)+\sqrt{\gamma_{1}} L_{1}(t)+\sqrt{\gamma_{2}} L_{2}(t) .
$$

These equations coincide with those obtained for the Markovian series product systems in the literatures (see, 
e.g., Ref. [9]). This concludes this remark.

The dynamical equation (33) can be extended readily to $n$ cascade-connected subsystems to obtain the following quantum stochastic differential equation

$$
\begin{aligned}
\dot{X}= & \sum_{l, r=1}^{n} \int_{0}^{t}\left\{\gamma_{l r}(t-\tau) L_{l}^{\dagger}(\tau)\left[X(t), L_{r}(t)\right]\right. \\
& \left.+\gamma_{l r}^{*}(t-\tau)\left[L_{r}^{\dagger}(t), X(t)\right] L_{l}(\tau)\right\} d \tau \\
& -i\left[X, H_{S}\right]+\sum_{l=1}^{n}\left\{\tilde{b}_{l, \text { in }}\left[L_{l}^{\dagger}, X\right]+\left[X, L_{l}\right] \tilde{b}_{l, \mathrm{in}}^{\dagger}\right\}
\end{aligned}
$$

where $\gamma_{l r}(t-\tilde{t})$ is defined by Eq. (32);

$$
H_{S}=\sum_{l=1}^{n} H_{l}+\sum_{l<r} H_{l r}
$$

$H_{l=1, \cdots, n}$ is the free Hamiltonian of the $l$-th subsystem; and $H_{l r}$ is the field-mediated interaction Hamiltonian

$$
H_{l r}=i \int_{0}^{t}\left[\gamma_{l r}^{\theta}(\tau-t) L_{r} L_{l}^{\dagger}(\tau)-\text { h.c. }\right] d \tau \text {. }
$$

The function $\gamma_{l r}^{\theta}(t-\tilde{t})$ is defined by

$$
\gamma_{l r}^{\theta}(t-\tilde{t})=\int_{-\infty}^{+\infty} \kappa_{l}^{*}(\tau-t) \kappa_{r}(\tau-\tilde{t}) \theta(t-\tau) d \tau,
$$

where $\theta(t)$ is the step function defined by Eq. (30). The output equation can be written as

$$
b_{\text {out }}(t)=b_{\text {in }}(t)+\sum_{l=1}^{n} \int_{0}^{t} \kappa_{l}(t-\tau) L_{l}(\tau) d \tau .
$$

Transforming this into the Schrödinger picture, we can obtain the following second-order master equation

$$
\dot{\rho}=-i\left[H_{S}, \rho\right]+\sum_{l, r=1}^{n} \int_{0}^{t}\left\{\gamma_{l r}(t-\tau)\left[L_{r} \rho(\tau), L_{\mathrm{H}_{S}, l}^{\dagger}(\tau-t)\right]+\gamma_{l r}^{*}(t-\tau)\left[L_{\mathrm{H}_{\mathrm{S}}, l}(\tau-t), \rho(\tau) L_{r}^{\dagger}\right]\right\} d \tau
$$

where

$$
L_{\mathrm{H}_{\mathrm{S}}, l}(t)=\exp \left(i H_{S} t\right) L_{l} \exp \left(-i H_{S} t\right)
$$

\section{B. Example: non-Markovian qubit networks in superconducting circuits}

As the first example, we apply our non-Markovian network formalism to superconducting circuits [4]. Here, as a simple example, we consider how to couple two distant single Cooper pair boxes (CPBs) by a microwave field. As shown in Fig. [5 to suppress the decoherence effects, we embed two CPBs into two superconducting transmission line resonators (TLRs). When we average over the degrees of freedom of the TLRs, the interactions between the CPBs and the input fields become non-Markovian. This can be understood by noting that the TLRs work as microwave cavities, and these act as low-pass filters. The white-noise input fields are filtered by the TLRs and changed into non-Markovian Lorentz noises, and these in turn interact with the CPBs. Thus, the qubit network considered here is a typical non-Markovian quantum network.

The Hamiltonian of the $j^{\text {th }} \mathrm{CPB}(j=1,2)$ can be represented as

$$
H_{\mathrm{CPB}, \mathrm{j}}=4 E_{C}\left(n_{j}-n_{g j}\right)^{2}-E_{J}\left(\Phi_{x j}\right) \cos \phi_{j},
$$

where $\phi_{j}$ denotes the phase drop across the $j^{\text {th }} \mathrm{CPB}$, with $n_{j}=-i \partial /\left(\partial \phi_{j}\right)$ as its conjugate operator. The operator $n_{j}$ represents the number of Cooper pairs on the island electrode. The scalar $n_{g j}$ is the reduced charge number on the control gate in units of Cooper pairs. This is given by $n_{g j}=-C_{g} V_{g j} / 2 e$, where $C_{g}$ and $V_{g j}$ are the gate capacitance and gate voltage of the $j^{t h}$ CPB. The scalar $E_{C}=e^{2} / 2\left(C_{g}+2 C_{J}^{0}\right)$ is the single-electron charging energy of a single $\mathrm{CPB}$ and $C_{J}^{0}$ is the capacitance of a single Josephson junction. The Josephson energy $E_{J}\left(\Phi_{x j}\right)$ of the $j^{\text {th }}$ DC superconducting quantum interference device (SQUID) can be calculated by

$$
E_{J}\left(\Phi_{x j}\right)=2 E_{J}^{0} \cos \left(\pi \frac{\Phi_{x j}}{\Phi_{0}}\right)
$$

where $E_{J}^{0}$ represents the Josephson energy of a single Josephson junction; $\Phi_{x j}$ is the external flux piercing the SQUID loop of the $j^{\text {th }} \mathrm{CPB}$; and $\Phi_{0}$ is the flux quantum. For simplicity, we assume that $E_{C}$ and $E_{J}^{0}$ are the same for each Josephson junction in the two CPBs.

Near the charge-degenerate point with $n_{g j}=0.5$, the two lowest-energy levels of the $j^{\text {th }} \mathrm{CPB}$ are close to each other and far separated from higher-energy levels. Thus, we can approximately consider a single CPB as a twolevel system. In the qubit basis, the Hamiltonian $H_{\mathrm{CPB}, \mathrm{j}}$ 


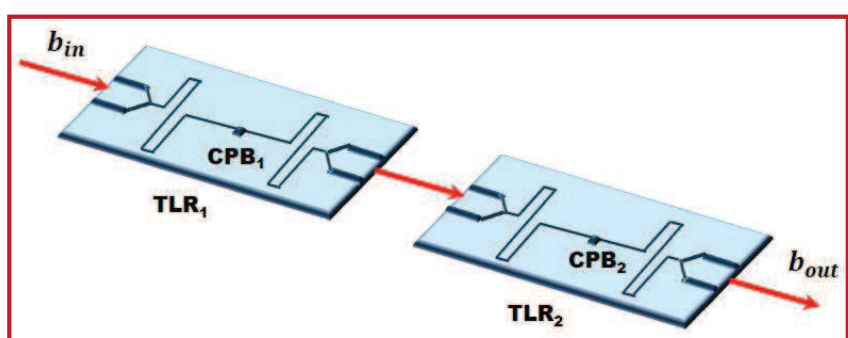

(a)

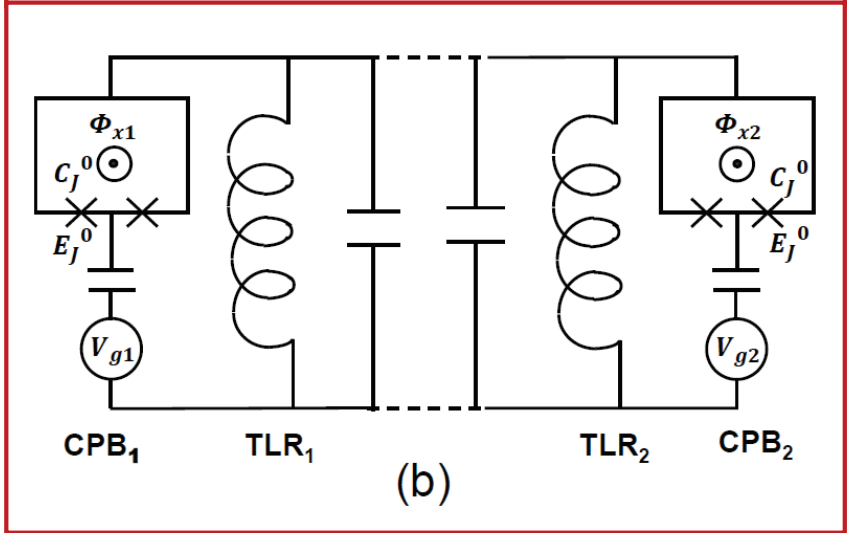

FIG. 5: (Color online) Schematic diagrams of (a) two cascadeconnected CPB-TLR (Cooper-pair box and transmission line resonator) input-output systems and (b) the equivalent superconducting circuits.

can be diagonalized as

$$
H_{\mathrm{CPB}, \mathrm{j}}=\frac{\tilde{\omega}_{q j}}{2} \sigma_{z}^{(j)},
$$

where $\tilde{\omega}_{q j}=\left(E_{C j}^{2}+E_{J j}^{2}\right)^{1 / 2}, E_{J j}=E_{J}\left(\Phi_{x j}\right)$ and $E_{C j}=4 E_{C}\left(1-2 n_{g j}\right)$.

As shown in Fig. [5], the $j^{\text {th }} \mathrm{CPB}$ is capacitively coupled to the $j^{\text {th }}$ TLR. The Hamiltonian of the $j^{\text {th }}$ coupled CPBTLR system can be written as

$$
\begin{aligned}
\tilde{H}_{j}= & g_{j}\left(-\cos \alpha_{j} \sigma_{z}^{(j)}+\sin \alpha_{j} \sigma_{x}^{(j)}\right)\left(a_{j}+a_{j}^{\dagger}\right) \\
& +\frac{\tilde{\omega}_{q j}}{2} \sigma_{z}^{(j)}+\omega_{c j} a_{j}^{\dagger} a_{j},
\end{aligned}
$$

where $a_{j}$ is the annihilation operator of the singlemode electromagnetic field in the $j^{\text {th }}$ TLR; $g=$ $-e\left(C_{g} / C_{\Sigma}\right) V_{\mathrm{rms}, \mathrm{j}}^{0}$ is the coupling strength between the resonator and the qubit; $C_{\Sigma}=C_{g}+2 C_{J}^{0}$ is the total capacitance of the SCB; $V_{\mathrm{rms}}^{0}=\sqrt{\omega_{c} / 2 C_{r}}$ is the root mean square (rms) of the voltage across the $L C$ circuit with $C_{r}$ representing the capacitance of the resonator; and $\alpha_{j}$ is defined by

$$
\alpha_{j}=\arctan \left[E_{J}\left(\phi_{x j}\right) / E_{C}\left(1-2 n_{g j}\right)\right] .
$$

Letting the SCBs work at the charge-degenerate point, such that $n_{g j}=1 / 2(j=1,2)$, and introducing the rotating wave approximation, we can obtain the following effective Hamiltonian of the $j^{\text {th }}$ coupled CPB-TLR system

$$
H_{j}=\frac{\omega_{q j}}{2} \sigma_{z}^{(j)}+\omega_{c j} a_{j}^{\dagger} a_{j}+g_{j}\left(a_{j}^{\dagger} \sigma_{-}^{(j)}+a_{j} \sigma_{+}^{(j)}\right),
$$

where $\omega_{q j}=E_{J}\left(\phi_{x j}\right)$.

The cavity mode in the $j^{\text {th }}$ TLR is coupled to a transmitting field in an auxiliary transmission line between two TLRs. The total Hamiltonian of the $j^{\text {th }}$ SCB-TLR system and the input field $b_{\mathrm{in}, \mathrm{j}}$ can be represented by

$$
\begin{aligned}
H_{\mathrm{tot}, \mathrm{j}}= & \frac{\omega_{q j}}{2} \sigma_{z}^{(j)}+\omega_{c j} a_{j}^{\dagger} a_{j}+g_{j}\left(a_{j}^{\dagger} \sigma_{-}^{(j)}+a_{j} \sigma_{+}^{(j)}\right) \\
& +i \sqrt{\gamma_{j}}\left(a_{j}^{\dagger} b_{\mathrm{in}, \mathrm{j}}-b_{\mathrm{in}, \mathrm{j}}^{\dagger} a_{j}\right) .
\end{aligned}
$$

where $\gamma_{j}$ is determined by the coupling between the cavity mode and the input field. To be concentrated on the dynamics of the qubit, we eliminate the degrees of freedom of the cavity mode. From Eq. (45), it can be shown that

$$
\dot{a}_{j}=-\left(i \omega_{c j}+\frac{\gamma_{j}}{2}\right) a_{j}+\sqrt{\gamma_{j}} b_{\mathrm{in}, \mathrm{j}}-i g_{j} \sigma_{-}^{(j)} .
$$

Let us now introduce the following weak coupling assumption

$$
\omega_{c j}, \gamma_{j} \gg g_{j}
$$

so we can omit the last term in Eq. (46) when we consider the dynamics of the cavity mode. Thus, we can now solve Eq. (46)

$$
a_{j}(t)=\sqrt{\gamma_{j}} \int_{0}^{t} \exp \left[-\left(i \omega_{c j}+\gamma_{j} / 2\right)(t-\tau)\right] b_{\mathrm{j}, \text { in }}(\tau) d \tau .
$$

Substituting Eq. (47) into the Hamiltonian $H_{\text {tot,j }}$ in Eq. (45), we can obtain an effective Hamiltonian to represent the coupling between the $j^{\text {th }}$ qubit and the effective input field

$$
\tilde{H}_{\mathrm{eff}, \mathrm{j}}=\frac{\omega_{q j}}{2} \sigma_{z}^{(j)}+i\left(\tilde{b}_{\mathrm{in}, \mathrm{j}}^{\dagger} \sigma_{-}^{(j)}-\sigma_{+}^{(j)} \tilde{b}_{\mathrm{in}, \mathrm{j}}\right),
$$

where

$$
\tilde{b}_{\mathrm{in}, \mathrm{j}}(t)=\int_{0}^{t} i g_{j} \sqrt{\gamma_{j}} e^{-\left(i \omega_{c j}+\gamma_{j} / 2\right)(t-\tau)} b_{\mathrm{in}, \mathrm{j}}(\tau) d \tau .
$$

If we additionally add an ac gate voltage $V_{g j}=$ $V_{0 j} \cos \left(\omega_{g j} t\right)$ on the gate of the $j^{\text {th }} \mathrm{CPB}$, where $V_{0 j}$ and $\omega_{g j}$ are the amplitude and frequency of the gate voltage, we can obtain the following effective Hamiltonian in the rotating frame

$$
\tilde{H}_{\mathrm{eff}, \mathrm{j}}=\frac{\Delta_{q j}}{2} \sigma_{z}^{(j)}+i\left(\tilde{b}_{\mathrm{in}, \mathrm{j}}^{\dagger} \sigma_{-}^{(j)}-\sigma_{+}^{(j)} \tilde{b}_{\mathrm{in}, \mathrm{j}}\right),
$$

under the condition that $C_{g} V_{0 j} E_{C} / 2 e \ll \Delta_{q j}=$ $E_{J}-\omega_{q j}$. By comparing Eq. (49) and Eq. (11), we can see that the $j^{\text {th }} \mathrm{CPB}$ is just directly coupled to the effective non-Markovian field $\tilde{b}_{\mathrm{in}, \mathrm{j}}$ with 
$\kappa_{j}(t)=i g_{j} \sqrt{\gamma_{j}} \exp \left[-\left(\omega_{c j}+\gamma_{j} / 2\right) t\right]$. Additionally we can see that the total system we consider here is just a cascade-connected two-qubit system mediated by a nonMarkovian field. If $b_{\mathrm{in}, \mathrm{j}}$ is a white noise, it can be easily verified that the spectrum of $\tilde{b}_{\mathrm{in}, \mathrm{j}}(t)$ is of Lorentz type. In fact, it can be calculated in the frequency domain that

$$
\begin{aligned}
\tilde{b}_{\mathrm{in}, \mathrm{j}}(\omega) & =\kappa_{j}(\omega) b_{\mathrm{in}, \mathrm{j}}(\omega) \\
& =\frac{i g_{j} \sqrt{\gamma_{j}}}{\gamma_{j} / 2+i\left(\omega-\omega_{c j}\right)} b_{\mathrm{in}, \mathrm{j}}(\omega),
\end{aligned}
$$

where $\tilde{b}_{\mathrm{in}, \mathrm{j}}(\omega), b_{\mathrm{in}, \mathrm{j}}(\omega), \kappa(\omega)$ are the Fourier transform of $b_{\mathrm{in}, \mathrm{j}}(t), b_{\mathrm{in}, \mathrm{j}}(t), \kappa(t)$. From Eq. (15), it can be shown that

$$
\tilde{\gamma}_{j}(\omega)=\left|\kappa_{j}(\omega)\right|^{2}=\frac{g_{j}^{2} \gamma_{j}}{\gamma_{j}^{2} / 4+\left(\omega-\omega_{c j}\right)^{2}} .
$$

Note that

$$
\begin{aligned}
{\left[\tilde{b}_{\mathrm{in}, \mathrm{j}}(t), \tilde{b}_{\mathrm{in}, \mathrm{j}}^{\dagger}(\tilde{t})\right] } & =\tilde{\gamma}_{j}(t-\tilde{t})=\int_{0}^{\infty} e^{-i \omega(t-\tilde{t})} \tilde{\gamma}_{j}(\omega) d \omega \\
& =g_{j}^{2} \exp \left[-\gamma_{j}|t-\tilde{t}| / 2\right] .
\end{aligned}
$$

We can see that $\tilde{b}_{\mathrm{in}, \mathrm{j}}(t)$ is a Lorentz-type noise.

To simplify our discussions, let us assume that the two qubits have the same system parameters, i.e., $\Delta_{q}=$ $\Delta_{q 1}=\Delta_{q 2}, g=g_{1}=g_{2}$, and $\gamma=\gamma_{1}=\gamma_{2}$. From Eq. (41), we can obtain the master equation of the nonMarkovian two-qubit system we consider here

$$
\begin{aligned}
\dot{\rho}= & -i\left[\frac{\Delta_{q}}{2} J_{z}+\alpha(t) \sigma_{-}^{(1)} \sigma_{+}^{(2)}+\alpha^{*}(t) \sigma_{+}^{(1)} \sigma_{-}^{(2)}, \rho\right] \\
& +\int_{0}^{t}\left\{\beta(t-\tau)\left[J_{-} \rho(\tau), J_{+}\right]+\text {h.c. }\right\} d \tau
\end{aligned}
$$

where $J_{\alpha=z, \pm}=\sigma_{\alpha}^{(1)}+\sigma_{\alpha}^{(2)}$ is the collective two-qubit operator; and $\alpha(t)$ and $\beta(t)$ can be calculated by

$$
\begin{aligned}
& \alpha(t)=\frac{i g^{2} \exp \left(i \Delta_{q} t\right)}{\gamma / 2+i \Delta_{q}}-\frac{i g^{2} \exp (-\gamma t / 2)}{\gamma / 2+i \Delta_{q}}, \\
& \beta(t)=g^{2} \exp \left[-\gamma t / 2+i \Delta_{q} t\right] .
\end{aligned}
$$

Additionally, if $\Delta_{q}=E_{J}-\omega_{q}=0$, we can rewrite Eq. (52) by introducing the first Markovian approximation, i.e., to replace $\rho(\tau)$ in the last integral term in Eq. (52) by $\rho(t)$, as

$$
\dot{\rho}=-i\left[\alpha(t) \sigma_{-}^{(1)} \sigma_{+}^{(2)}+\text { h.c., } \rho\right]+\Gamma(t) \mathcal{D}\left[J_{-}\right] \rho,
$$

where

$$
\alpha(t)=i \Gamma(t)=i \frac{2 g^{2}}{\gamma}\left(1-e^{-\gamma t / 2}\right)
$$

and the superoperator $\mathcal{D}\left[J_{-}\right] \rho$ is defined by

$$
D\left[J_{-}\right] \rho=J_{-} \rho J_{+}-\frac{1}{2} J_{+} J_{-} \rho-\frac{1}{2} \rho J_{+} J_{-} .
$$

It can be verified that both $\alpha(t)$ and $\Gamma(t)$ decrease when $\gamma$ decreases. This means that both the coherent interaction between the two qubits and the damping induced by the transmitting field decrease with the increase of the correlation time of the non-Markovian Lorentz noises $\tilde{b}_{\text {in,j }}(t)$ scaled by $1 / \gamma$.

In Fig. 6] we show the evolution of the concurrence of the two qubits. The concurrence is defined by

$$
C(\rho)=\max \left\{\lambda_{1}-\lambda_{2}-\lambda_{3}-\lambda_{4}, 0\right\} .
$$

where $\rho$ is the system density matrix given by Eq. (54); $\lambda_{i}$ 's are the square roots of the eigenvalues, in a decreasing order, of the matrix $\rho$; and $\rho^{*}$ is the complex conjugate of $\rho$. From Fig. 6 we see that the damping rate of the concurrence decreases as the bath coupling strength $g$ decreases, but increases when the correlation time of the environment $\left(\tau_{\text {env }}=1 / \gamma\right)$ increases. This means that in non-Markovian environments the two-qubit entanglement is preserved longer than in a Markovian environment, which agrees with our intuition.

The two-qubit dynamics given by Eq. (52) can be extended to multi-qubit networks to study many-body physical phenomena, such as quantum entanglement and correlations.

\section{Example: feedforward and feedback control network}

In this subsection, we consider using networks for quantum control [50 55]. A simple control network is composed of two cascade-connected subsystems. One is called the controller, and the other the plant. The purpose of the network is to improve the performance of the plant by connecting it to the controller. Whether such a strategy will work depends in general upon the properties of each. Such a configuration is useful if for some physical reason the controller can be tailored in ways that the plant cannot.

It is common to divide control networks into two classes. The first one is an open-loop or "feedforward" scenario in which the input field is first fed into the controller to obtain a control signal and then the control signal is fed into the quantum plant to control the dynamics of the plant (see Fig. 7). In this method, the control signal contains no prior information of the system dynamics. The second one is a feedback control network [30 49]. This is also called closed-loop control, in which the input field is first fed into the plant to extract the information we need. The information-bearing output field is then fed into the quantum controller to obtain an output control signal which is fed back to change the dynamics of the plant. Such a control system may be viewed as a threepartite quantum cascade system: plant-controller-plant. Since the signal fed into the controller contains real-time information about the state of the plant, we can use it to adjust the behavior of the controller. 

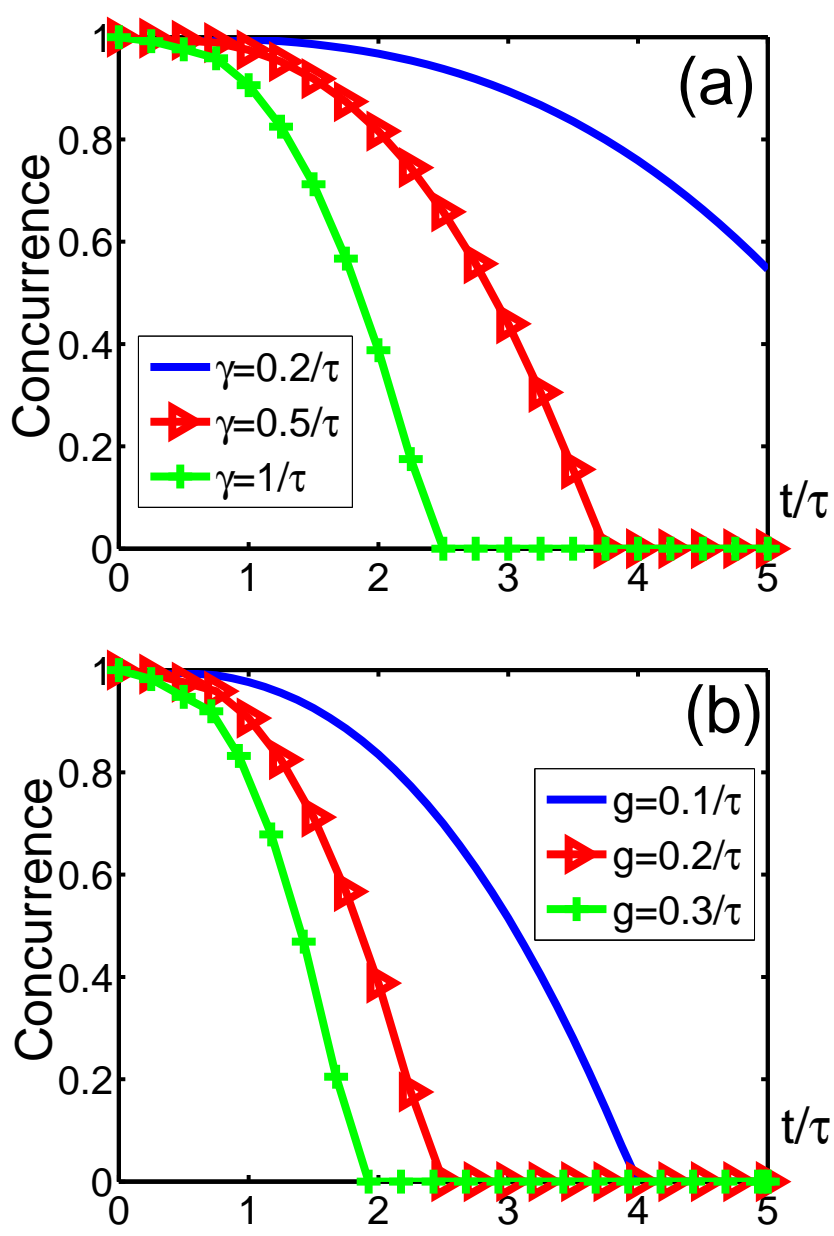

FIG. 6: (color online) Evolution of the concurrence (a) for various non-Markovian correlation rates $\gamma$ (the blue curve, red triangle curve, and the green plus-sign curve correspond to $\gamma=0.2 / \tau, 0.5 / \tau, 1 / \tau$ ) and (b) for various coupling strengths $g$ (the blue curve, red triangle curve, and the green plus-sign curve correspond to $g=0.1 / \tau, 0.2 / \tau, 0.3 / \tau)$. Here $\tau=10$ ns. The decay of the concurrence speeds up when increasing the correlation rate $\gamma$ of the non-Markovian noises and the qubit-environment coupling strength $g$.

By examining Eq. (39) one can see that the quantum controller introduces Hamiltonian terms like

$$
H_{c}=\left\{-i\left[\int_{0}^{t} \gamma(t-\tau) u_{c}(\tau) d \tau\right] L+\text { h.c. }\right\}
$$

where $u_{c}(t)=u_{c}\left(X_{c, 1}(t), \cdots, X_{c, n}(t)\right)$ is a function of the system variables $X_{c, 1}(t), \cdots, X_{c, n}(t)$ of the quantum controller and $L$ is the system operator of the plant. The signal $u_{c}(t)$ is the control signal that is input to the plant. The idea is to design the controller dynamics so that the evolutions of $X_{c, 1}(t), \cdots, X_{c, n}(t)$ generate the appropriate control signal, $u_{c}(t)$. The primary difference between feedforward (open-loop) and feedback (closedloop) controls is that in the former the controller variables $X_{c, 1}, \cdots, X_{c, n}$ can be looked as extrogenous vari-

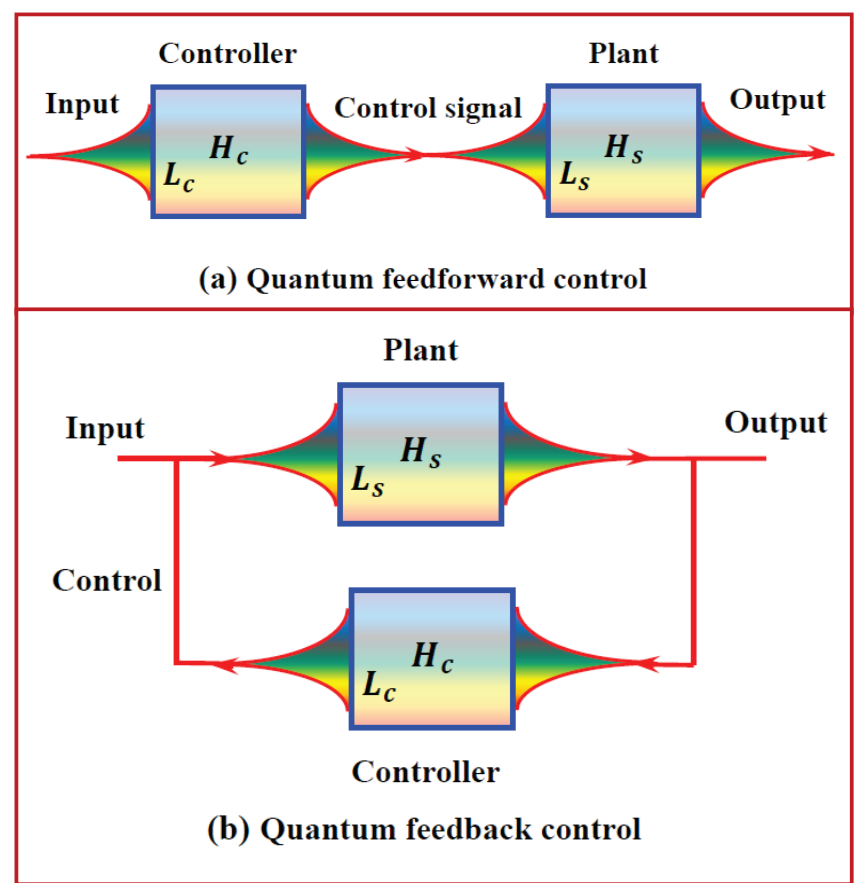

FIG. 7: (Color online) Schematic diagrams of (a) quantum feedforward control network and (b) quantum feedback control network. Here, $H_{c}\left(H_{s}\right)$ and $L_{c}\left(L_{s}\right)$ are the Hamiltonian and dissipation operator for the controller (plant).

ables that do not depend on the dynamics of the plant. In the latter, $X_{c, 1}, \cdots, X_{c, n}$ are functions of the endogeous variables of the plant, and can be obtained in terms of the plant variables by solving the dynamical equation of the controller. These two control methods have different advantages, and the best performance might only be achieved by combing them.

For a concrete example, let us choose the plant to be a single-mode cavity so that

$$
H_{s}=\omega_{a} a^{\dagger} a, \quad L_{s}=a,
$$

and the controller to be a fully-controllable two-qubit system. The Hamiltonian and dissipation operator of the controller are

$$
H_{c}=\frac{\Delta_{q}}{2} \sigma_{z}+\mu_{d}^{*} \sigma_{-}+\mu_{d} \sigma_{+}, \quad L_{c}=\sigma_{-} .
$$

Here $\Delta_{q}$ is the detuning between the transition frequency of the qubit and the frequency of the extrogenous driving field and $\mu_{d}$ is a classical extrogenous control parameter.

We now show what happens if we give the controller a fast damping rate, so that it adjusts very quickly to change in the plant. In this case we can average out the degrees of freedom of the controller (see the derivations in Appendix B). For the case of feedforward control, the reduced Hamiltonian of the cavity can be written as

$$
H_{1, \mathrm{eff}}=\omega_{a} a^{\dagger} a+\left[u_{c}^{*}(t) a+u_{c}(t) a^{\dagger}\right],
$$




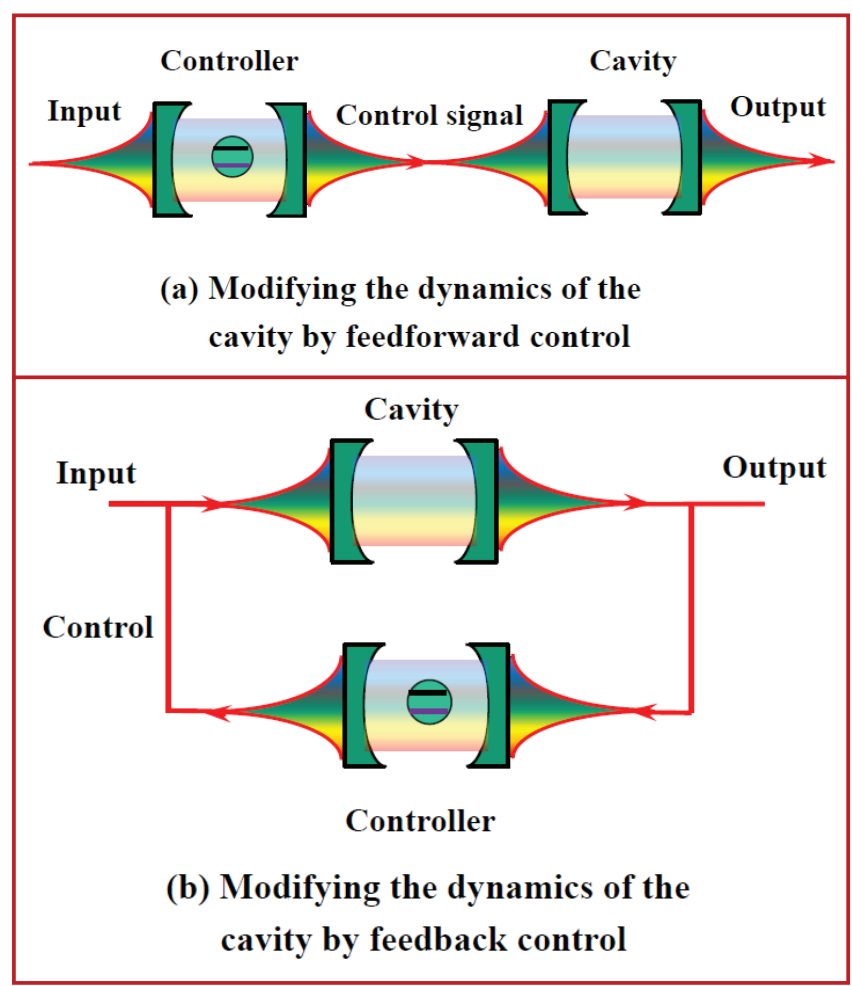

FIG. 8: (Color online) Modifying the dynamics of a cavity using a qubit inside the controller by (a) feedforward control and (b) feedback control.

where $u_{c}(t)$ is a classical control parameter depending on the state of the qubit but does not depend on the dynamics of the controlled cavity mode $a$. This is simply the Hamiltonian of a driven harmonic oscillator. As a comparison, for feedback control the reduced Hamiltonian of the cavity becomes

$H_{2, \mathrm{eff}}=\omega_{a} a^{\dagger} a+\left\{\left[\int_{0}^{t} \gamma^{*}(t-\tau) a^{\dagger}(\tau) d \tau\right] a(t)+\right.$ h.c. $\}$.

Non-classical optical effects such as squeezing induced by the memory term in Eq. (59) might well be observable in this case [56]. We see that feedback control can generate a class of evolutions that are impossible with an openloop connection.

\section{CONCLUSIONS}

In summary, we have extended quantum input-output theory to arbitrary non-Markovian networks of systems connected via continuous-wave fields. We have derived the Heisenberg picture quantum stochastic differential equation for the systems in the network, the corresponding perturbative master equations, and all the input-output relations. We have applied this general formalism to a model of two superconducting charge qubits intracting via a cascade connection. We showed that this system was non-Markovian because the cavities with which the qubits connect to each other act as filters for the quantum noise. For this system we analyzed the dynamics of the entanglement between the qubits, and showed that it was affected by the non-Markovian nature of the network. We also use our model to analyze the difference between feedforward and feedback networks in which the controller has a fast response time. It is clear from our analysis that non-Markovian effects can have a significant effect on the behavior of mesoscopic quantum networks, and the analysis of these effects may be important for future quantum devices.

\section{ACKNOWLEDGMENTS}

J. Zhang would like to thank Prof. W.-M. Zhang for helpful discussions. J. Zhang and R. B. Wu are supported by the National Natural Science Foundation of China under Grant Nos. 61174084, 61134008, 60904034. Y.-X. Liu is supported by the National Natural Science Foundation of China under Grant Nos. 10975080, 61025022 . K. Jacobs is partially supported by the NSF under Project Nos. PHY-0902906, and PHY-1005571, and the ARO MURI grant W911NF-11-1-0268. F. Nori is partially supported by the ARO, JSPS-RFBR contract No. 12-02-92100, Grant-in-Aid for Scientific Research (S), MEXT Kakenhi on Quantum Cybernetics, and the JSPS via its FIRST program.

\section{Appendix A: Derivations of the non-Markovian dynamical and output equations}

From the system Hamiltonian (1), we can obtain the Heisenberg equation of an arbitrary system operator $X$

$$
\begin{aligned}
\dot{X}= & -i\left[X, H_{\mathrm{sys}}\right]+\int d \omega\left\{\kappa(\omega) b^{\dagger}(\omega, t)[X, L]\right. \\
& \left.-\kappa^{*}(\omega)\left[X, L^{\dagger}\right] b(\omega, t)\right\},
\end{aligned}
$$

and the equation of the bath operator $b(\omega)$

$$
\dot{b}(\omega, t)=-i \omega b(\omega, t)+\kappa(\omega) L .
$$

We can solve Eq. (A2) and obtain

$$
b(\omega, t)=e^{-i \omega t} b(\omega)+\kappa(\omega) \int_{0}^{t} e^{-i \omega(t-\tau)} L(\tau) d \tau,
$$

where $b(\omega)=b(\omega, 0)$ is the initial condition of $b(\omega, t)$. Similarly,

$$
b(\omega, t)=e^{-i \omega\left(t-t_{1}\right)} b\left(\omega, t_{1}\right)-\kappa(\omega) \int_{t}^{t_{1}} e^{-i \omega(t-\tau)} L(\tau) d \tau,
$$


where $t_{1} \geq t$. The input and output fields $b_{\text {in }}(t)$ and $b_{\text {out }}(t)$ are defined as the Fourier transform of $b(\omega)$ and $b\left(\omega, t_{1}\right)$ respectively

$$
\begin{aligned}
b_{\text {in }}(t) & =\frac{1}{\sqrt{2 \pi}} \int_{-\infty}^{+\infty} b(\omega) e^{-i \omega t} d t \\
b_{\text {out }}(t) & =\frac{1}{\sqrt{2 \pi}} \int_{-\infty}^{+\infty} b\left(\omega, t_{1}\right) e^{-i \omega\left(t-t_{1}\right)} d t .
\end{aligned}
$$

From Eqs. (A3) and (A4), we have

$$
b_{\text {out }}(t)=b_{\text {in }}(t)+\int_{0}^{t_{1}} \kappa(t-\tau) L(\tau) d \tau .
$$

Let $t_{1} \rightarrow t$, we can obtain the output equation (17).

Furthermore, using the identities

$$
\begin{aligned}
\tilde{b}_{\text {in }} & =\int_{-\infty}^{+\infty} \kappa(t-\tau) b_{\text {in }}(\tau) d \tau \\
& =\int_{-\infty}^{+\infty} \kappa(\omega) e^{-i \omega t} b(\omega) d \omega
\end{aligned}
$$

and

$$
\begin{aligned}
\gamma(t-\tilde{t}) & =\int_{-\infty}^{+\infty} \kappa^{*}(t-\tau) \kappa(\tilde{t}-\tau) d \tau \\
& =\int_{-\infty}^{+\infty} \kappa(\omega) \kappa^{*}(\omega) e^{-i \omega(\tilde{t}-t)} d \omega
\end{aligned}
$$

we can obtain Eq. (16) by substituting Eq. (A3) into Eq. A1).

To derive the master equation (18), we first change into the interaction picture, in which the effective Hamiltonian $H_{\text {eff }}$ can be rewritten as

$$
H_{\mathrm{I}, \mathrm{eff}}=i\left[b_{\text {in }}^{\dagger}(t) L_{\mathrm{H}_{\mathrm{S}}}(t)-L_{\mathrm{H}_{\mathrm{S}}}(t) b_{\mathrm{in}}(t)\right],
$$

where $L_{\mathrm{H}_{\mathrm{S}}}(t)$ is given in Eq. (19). The density operator $\rho_{\mathrm{I}, \text { tot }}$ satisfies the following Liouville equation

$$
\dot{\rho}_{\mathrm{I}, \mathrm{tot}}=-i\left[H_{\mathrm{I}, \mathrm{eff}}(t), \rho_{\mathrm{I}, \mathrm{tot}}\right] .
$$

Integrating the two sides of Eq. (A7), we have

$$
\rho_{\mathrm{I}, \mathrm{tot}}(t)=-i \int_{0}^{t}\left[H_{\mathrm{I}, \mathrm{eff}}(\tau), \rho_{\mathrm{I}, \mathrm{eff}}(\tau)\right] d \tau .
$$

Substituting Eq. (A8) into Eq. (A7), we can obtain

$$
\dot{\rho}_{\mathrm{I}, \mathrm{tot}}=\int_{0}^{t}\left[H_{\mathrm{I}, \mathrm{eff}}(t),\left[H_{\mathrm{I}, \mathrm{eff}}(\tau), \rho_{\mathrm{I}, \mathrm{tot}}(\tau)\right]\right] d \tau .
$$

Tracing over the degrees of freedom of the input field, we can obtain the dynamical equation of the system density operator $\rho_{I}=\operatorname{tr}_{B} \rho_{\mathrm{I}, \text { tot }}$

$$
\dot{\rho}_{I}=\int_{0}^{t} \operatorname{tr}_{B}\left\{\left[H_{\mathrm{I}, \mathrm{eff}}(t),\left[H_{\mathrm{I}, \mathrm{eff}}(\tau), \rho_{\mathrm{I}, \mathrm{tot}}(\tau)\right]\right]\right\} d \tau \text {. }
$$

Let us then introduce the Born approximation and assume that the input field stays in the vacuum state, we have

$$
\rho_{\mathrm{I}, \text { tot }}(t)=\rho_{I}(t) \otimes|0\rangle_{B B}\langle 0| .
$$

Notice that it can be shown that

$$
\begin{gathered}
\left\langle b_{\text {in }}(t) b_{\text {in }}^{\dagger}(\tilde{t})\right\rangle=\delta(t-\tilde{t}), \\
\left\langle b_{\text {in }}^{\dagger}(t) b_{\text {in }}(\tilde{t})\right\rangle=\left\langle b_{\text {in }}^{\dagger}(t) b_{\text {in }}^{\dagger}(\tilde{t})\right\rangle=\left\langle b_{\text {in }}(t) b_{\text {in }}(\tilde{t})\right\rangle=0,
\end{gathered}
$$

where $\langle\cdot\rangle$ is defined by $\langle R\rangle=\langle 0|b| 0\rangle_{B}$. Substituting Eqs. (A6), (A11), and (A12) into Eq. (A10), we can verify that

$$
\dot{\rho}_{I}=\int_{0}^{t}\left\{\gamma(t-\tau)\left[L_{H_{S}}(t) \rho_{I}(\tau), L_{H_{S}}^{\dagger}(\tau)\right]+\text { h.c. }\right\} .
$$

We can derive Eq. (18) by transforming Eq. (A13) back into the Schrödinger picture.

\section{Appendix B: Derivations of the effective Hamiltonians for feedforward and feedback control}

For the case of feedforward control, the dynamics of the feedforward control system shown in Fig. 8)(a) can be represented by

$$
\begin{aligned}
\dot{\sigma}_{-} & =-i \Delta_{q} \sigma_{-}+i\left(\mu_{d}+g_{q b} b\right) \sigma_{z}, \\
\dot{\sigma}_{z} & =2 i\left(\mu_{d}+g_{q b} b\right) \sigma_{-}-2 i\left(\mu_{d}+g_{q b} b\right) \sigma_{+}, \\
\dot{b} & =-\frac{\gamma_{b}}{2} b-i \omega_{b} b+i g_{q b} \sigma_{-}+\sqrt{\gamma_{b}} b_{\mathrm{in}}, \\
b_{\mathrm{out}} & =b_{\mathrm{in}}+\sqrt{\gamma_{b}} b \\
\dot{a} & =-\left(\frac{\gamma_{a}}{2}+i \omega_{a}\right) a+\sqrt{\gamma_{a}} b_{\text {out }},
\end{aligned}
$$

where $b$ is the annihilation operator of the cavity mode directly interacting with the qubit; $\gamma_{a}, \gamma_{b}$ are the decay rates of the cavity modes $a, b$; and $g_{q b}$ is the coupling strength between the qubit and the cavity mode $b$. Here we omit the decay of the qubit. By averaging out the input noise, we have

$$
b=i g_{q b} \int_{0}^{t} \exp \left[-\left(i \omega_{b}+\gamma_{b} / 2\right)(t-\tau)\right] \sigma_{-}(\tau) d \tau .
$$

Under the condition that $\mu_{d} \gg g_{q b}\langle b\rangle$ where $\langle b\rangle$ is the average of the cavity mode $b$, we can rewrite Eq. (B1) by substituting Eq. (B2) into Eq. (B1)

$$
\begin{aligned}
\dot{\sigma}_{-}= & -i \Delta_{q} \sigma_{-}+i \mu_{d} \sigma_{z} \\
\dot{\sigma}_{z}= & 2 i \mu_{d} \sigma_{-}-2 i \mu_{d} \sigma_{+} \\
b_{\text {out }}= & b_{\text {in }}+i \sqrt{\gamma_{b}} g_{q b} \int_{0}^{t} e^{-\left(i \omega_{b}+\gamma_{b} / 2\right)(t-\tau)} \sigma_{-}(\tau) d \tau \\
\dot{a}= & i \sqrt{\gamma_{a} \gamma_{b}} g_{q b} \int_{0}^{t} e^{-\left(i \omega_{b}+\gamma_{b} / 2\right)(t-\tau)} \sigma_{-}(\tau) d \tau \\
& -\left(\gamma_{a} / 2+i \omega_{a}\right) a .
\end{aligned}
$$


Thus the dynamics of the cavity mode $a$ is dominated by

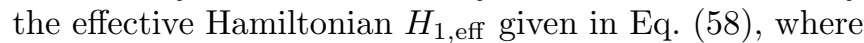

$u_{c}=-\sqrt{\gamma_{a} \gamma_{b}} g_{q b} \int_{0}^{t} \exp \left[-\left(i \omega_{b}+\gamma_{b} / 2\right)(t-\tau)\right] \sigma_{-}(\tau) d \tau$.

Under the semiclassical approximation, $\sigma_{-}(t)$ can be replaced by its average. With this simplification, $u_{c}(t)$ can be seen as a classical extrogenous control parameter because the dynamics of the qubit determined by $\sigma_{-}$and $\sigma_{z}$ does not depend on the cavity mode $a$. With similar discussions, we can also obtain the effective Hamiltonian $H_{2, \text { eff }}$ induced by feedback control given in Eq. (59).
[1] H. J. Kimble, Nature 453, 1023 (2008); D. Felinto, C. W. Chou, J. Laurat, E. W. Schomburg, H. de Riedmatte, and H. J. Kimble, Nature Phys. 2, 844 (2006); C.-W. Chou, J. Laurat, H. Deng, K. S. Choi, H. de Riedmatten, D. Felinto, and H. J. Kimble, Science 316, 1316 (2007).

[2] J. L. O'Brien, A. Furusawa, and J. Vučković, Nat. Photonics 3, 687 (2009).

[3] C. Ospelkaus, U. Warring, Y. Colombe, K. R. Brown, J. M. Amini, D. Leibfried, and D. J. Wineland, Nature 476, 181 (2011); J. T. Barreiro, M. Müller, P. Schindler, D. Nigg, T. Monz, M. Chwalla, M. Hennrich, C. F. Roos, P. Zoller, and R. Blatt, Nature 470, 486 (2011).

[4] J. Q. You and F. Nori, Physics Today 58 (11), 42 (2005); Nature 474, 589 (2011).

[5] P. D. Nation, J. R. Johansson, M. P. Blencowe, F. Nori, Rev. Mod. Phys. 84, 1 (2012); I. Buluta, F. Nori, Science 326, 108 (2009).

[6] C. W. Gardiner and M. J. Collett, Phys. Rev. A 31, 3761 (1985); C. W. Gardiner and P. Zoller, Quantum Noise (Springer-Verlag, Berlin, 2004) (3rd edition).

[7] C. W. Gardiner, Phys. Rev. Lett. 70, 2269 (1993); H. J. Carmichael, Phys. Rev. Lett. 70, 2273 (1993).

[8] L. Diosi, Phys. Rev. A 85, 034101 (2012).

[9] J. Gough and M. R. James, IEEE Trans. Automat. Contr. 54, 2530 (2009); H. Mabuchi, Phys. Rev. A 78, 032323 (2008).

[10] H. I. Nurdin, M. R. James, and A. C. Doherty, SIAM J. Control Optim. 48, 2686 (2009).

[11] G. F. Zhang and M. R. James, IEEE Trans. Automat. Contr. 56, 1535 (2011).

[12] R. I. Hudson and K. R. Parthasarathy, Commun. Math. Phys. 93, 301 (1984).

[13] M. Yanagisawa and H. Kimura, IEEE Trans. Automat. Contr. 48, 2107 (2003); ibid. 48, 2121 (2003).

[14] J. E. Gough, M. R. James, and H. I. Nurdin, Phys. Rev. A 81, 023804 (2010).

[15] A. Nazir, D.P.S. McCutcheon, and A. W. Chin, Phys. Rev. B 85, 224301 (2012).

[16] S. B. Xue, J. Zhang, R. B. Wu, C. W. Li, T. J. Tarn, J. Phys. B 44, 154016 (2011).

[17] W. Cui, Z. R. Xi, and Y. Pan, Phys. Rev. A 77, 032117 (2008).

[18] H. M. Wiseman and J. M. Gambetta, Phys. Rev. Lett. 101, 140401 (2008); T. A. Wheatley, D. W. Berry, H. Yonezawa, D. Nakane, H. Arao, D. T. Pope, T. C. Ralph, H. M. Wiseman, A. Furusawa, and E. H. Huntington, Phys. Rev. Lett. 104, 093601 (2010).

[19] D. J. Reilly, J. M. Taylor, J. R. Petta, C. M. Marcus, M. P. Hanson, A. C. Gossard, Science 321, 718 (2008); J. Medford, L. Cywinski, C. Barthel, C. M. Marcus, M. P. Hanson, A. C. Gossard, Phys. Rev. Lett. 108, 086802
(2012).

[20] R. McDermott, IEEE Trans. Appl. Supercond. 19, 2 (2009); S. Sendelbach, D. Hover, M. Mueck, and R. McDermott, Phys. Rev. Lett. 103, 117001 (2009).

[21] L. G. Remus, M. P. Blencowe, Y. Tanaka, Phys. Rev. B 80, 174103 (2009).

[22] H. P. Breuer and F. Petruccione, The Theory of Open Quantum Systems (Oxford University Press, Oxford, 2002).

[23] W. T. Strunz, L. Diosi, and N. Gisin, Phys. Rev. Lett. 82, 1801 (1999); J. Jing and T. Yu, Phys. Rev. Lett. 105, 240403 (2010); J. Jing, L.-A. Wu, J. Q. You, and T. Yu, Phys. Rev. A 85, 032123 (2012).

[24] I. Georgescu and F. Nori, Phys. World 25, 16 (2012); I.Buluta, S. Ashhab, and F. Nori, Rep. Prog. Phys. 74, 104401 (2011).

[25] V. Giovannetti, D. Vitali, Phys. Rev. A 63, 023812 (2001); S. Mancini, Phys. Rev. A 73, 010304(R) (2006).

[26] K. Jacobs, I. Tittonen, H. M. Wiseman, S. Schiller, Phys. Rev. A 60, 538 (1999).

[27] M.-H. Wu, C. U. Lei, W.-M. Zhang, and H.-N. Xiong, Opt. Exp. 18, 18407 (2010); H. T. Tan and W.-M. Zhang, Phys. Rev. A 83, 062310 (2011).

[28] J.-Q. Liao, Z. R. Gong, L. Zhou, Y.-X. Liu, C. P. Sun, and F. Nori, Phys. Rev. A 81, 042304 (2010); L. Zhou, S. Yang, Y.-X. Liu, C. P. Sun, and F. Nori, Phys. Rev. A 80, 062109 (2009).

[29] D. I. Tsomokos, S. Ashhab, F. Nori, Phys. Rev. A 82, 052311 (2010); P. Bertet, F. R. Ong, M. Boissonneault, A. Bolduc, F. Mallet, A. C. Doherty, A. Blais, D. Vion, and D. Esteve, book chapter in "Fluctuating Nonlinear Oscillators" by Oxford University Press, edited by Mark Dykman, e-print arXiv:1111.0501 v1; M. Leib, F. Deppe, A. Marx, R. Gross, M. Hartmann, arXiv:1202.3240 2.

[30] K. Jacobs and A. J. Landahl, Phys. Rev. Lett. 103, 067201 (2009).

[31] H. Mabuchi, Appl. Phys. Lett. 98, 193109 (2011).

[32] J. Zhang, R. B. Wu, C. W. Li, and T. J. Tarn, IEEE Trans. Automat. Contr. 55, 619 (2010); J. Zhang, Y.-X. Liu, R.-B. Wu, C.-W Li, and T.-J. Tarn, Phys. Rev. A 82, 022101 (2010); J. Zhang, R. B. Wu, Y.-X. Liu, C. W. Li, and T. J. Tarn, IEEE Trans. Automat. Contr. 57, 1997 (2012).

[33] S. Lloyd, Phys. Rev. A 62, 022108 (2000).

[34] H. Mabuchi, A. C. Doherty, Science 298, 1372 (2002); J. Kerckhoff, H. I. Nurdin, D. S. Pavlichin, and H. Mabuchi, Phys. Rev. Lett. 105, 040502 (2010).

[35] H. M. Wiseman and G. J. Milburn, Phys. Rev. Lett. 70, 548 (1993); Phys. Rev. A 49, 1350 (1994).

[36] A. C. Doherty and K. Jacobs, Phys. Rev. A 60, 2700 (1999); A. C. Doherty, S. Habib, K. Jacobs, H. Mabuchi, 
and S. M. Tan, Phys. Rev. A 62, 012105 (2000).

[37] V. P. Belavkin, J. Multivariate Anal. 42, 171 (1992); Commun. Math. Phys. 146, 611 (1992).

[38] D. A. Steck, K. Jacobs, H. Mabuchi, T. Bhattacharya, and S. Habib, Phys. Rev. Lett. 92, 223004 (2004).

[39] Z. H. Yan, X. J. Jia, X. L. Su, Z. Y. Duan, C. D. Xie, and K. C. Peng, Phys. Rev. A 85, 040305 (2012).

[40] K. De Greve, P. L. McMahon, D. Press, T. D. Ladd, D. Bisping, C. Schneider, M. Kamp, L. Worschech, S. Höling, A. Forchel, and Y. Yamamoto, Nat. Phys. 7, 872 (2011).

[41] M. S. Grinolds, P. Maletinsky, S. Hong, M. D. Lukin, R. L.Walsworth, and A. Yacoby, Nat. Phys. 7, 687 (2011).

[42] R. Ruskov, A. N. Korotkov, and K. Molmer, Phys. Rev. Lett. 105, 100506 (2010); Q. Zhang, R. Ruskov, and A. N. Korotkov, Phys. Rev. B 72, 245322 (2005).

[43] T. Brandes, Phys. Rev. Lett.105, 060602 (2010).

[44] A. Hopkins, K. Jacobs, S. Habib, and K. Schwab, Phys. Rev. B 68, 235328 (2003).

[45] M. J. Woolley, A. C. Doherty, and G. J. Milburn, Phys. Rev. B 82, 094511 (2010); G. J. Milburn and M. J. Woolley, Acta Phys. Slovaca 61, 486 (2011).

[46] Z. Liu, L. L. Kuang, K. Hu, L. T. Xu, S. H. Wei, L. Z. Guo, and X. Q. Li, Phys. Rev. A 82, 032335 (2010).

[47] J. Zhang, Y. X. Liu, and F. Nori, Phys. Rev. A 79, 052102 (2009).

[48] A detailed and pedagogical derivation of the input-output formalism can be found in: K. Jacobs, PhD dissertation, Imperial, London, Eprint arXiv:quant-ph/9810015.

[49] H. M. Wiseman and A. C. Doherty, Phys. Rev. Lett. 94, 070405 (2005); A. Chia, H. M. Wiseman, Phys. Rev. A 84, 012120 (2011).

[50] D. D Alessandro, Introduction to Quantum Control and Dynamics (Boca Raton, LA: Chapman and Hall, 2007).
[51] H. M. Wiseman and G. J. Milburn, Quantum Measurement and Control (Cambridge University Press, Cambridge, 2009).

[52] H. Mabuchi and N. Khaneja, Int. J. Robust Nonlinear Control 15, 647 (2005); C. Brif and R. Chakrabarti and H. Rabitz, New J. Phys. 12, 075008 (2010); D. Y. Dong and I. R. Petersen, IET Control Theory and Applications 4, 2651 (2010); C. Altafini and F. Ticozzi, IEEE Trans. Automat. Contr. 57, 1898 (2012).

[53] D. Y. Dong and I. R. Petersen, Automatica 48, 725 (2012); B. Qi, L. Guo, Syst. Contr. Lett. 59, 333 (2010); S. C. Hou, X. L. Huang, and X. X. Yi, Phys. Rev. A 82, 012336 (2010); T. L. Vu, S. S. Ge, and C. C. Hang, Phys. Rev. A 85, 012332 (2012).

[54] K. Y. Xia, M. Macovei, and J. Evers, Phys. Rev. B 84, 184510 (2011); J. Ma, X. G. Wang, C. P. Sun, F. Nori, Phys. Rep. 509, 89 (2011); A. F. Alharbi and Z. Ficek, Phys. Rev. A 82, 054103 (2010); X. Y. Lu, J. Wu, L. L. Zheng, and P. Huang, Opt. Commun. 283, 5279 (2010).

[55] B. Hwang and H.-S. Goan, Phys. Rev. A 85, 032321 (2012); N. Yamamoto, Phys. Rev. A 74, 032107 (2006); G. Tajimi and N. Yamamoto, Phys. Rev. A 85, 022303 (2012); M. Sarovar, H.-S. Goan, T. P. Spiller, and G. J. Milburn, Phys. Rev. A 72, 062327 (2005); Y. S. Greenberg, E. Il'ichev, and F. Nori, Phys. Rev. B 80, 214423 (2009); K. Jähne, C. Genes, K. Hammerer, M. Wallquist, E. S. Polzik, and P. Zoller, Phys. Rev. A 79, 063819 (2009); S. De Liberato, N. Lambert, and F. Nori, Phys. Rev. A 83, 033809 (2011).

[56] J. E. Gough and S. Wildfeuer, Phys. Rev. A 80, 042107 (2009); S. Iida, M. Yukawa, H. Yonezawa, N. Yamamoto, and A. Furusawa, IEEE Trans. Automat. Contr. 57, 2045 (2012). 